\title{
LA EVOLUCIÓN DE LOS SIGNIFICADOS DE LOS CONCEPTOS CIENTÍFICOS EN RELACIÓN CON LA ESTRUCTURA COGNITIVA DE LOS ESTUDIANTES
}

\author{
The evolution of scientific concepts \\ in relation to students' cognitive structure
}

\author{
Leonor Colombo de Cudmani ${ }^{1}$ \\ Marta A. Pesa ${ }^{2}$
}

\begin{abstract}
Resumen: El significado de los conceptos científicos constituye una interesante cuestión, tanto en el educación en ciencias como en la investigación y plantea interrogantes desde los más diversos ángulos: lógico, epistemológico, psicológico, lingüístico etc. El problema lógico y epistemológico, así como el proceso cognitivo de su construcción, tiene particular importancia en la enseñanza de la ciencia. En este trabajo nos proponemos analizar de la teoría del significado de Mario Bunge, desde lo epistemológico y la de Gérard Vergnaud, desde la psicología cognitiva. Estableceremos algunas relaciones con la visión epistemológica de la complejidad y propondremos algunas implicancias que se desprenden de este análisis para la enseñanza y el aprendizaje de la Ciencia y de la Física en particular.
\end{abstract}

Palabras clave: Evolución de conceptos científicos. Estructura cognitiva. Enseñanza de las Ciencias. Enseñanza de la Física.

\begin{abstract}
Scientific concepts are a very interesting issue in science education as well as in scientific research and suggest inquiries from diverse fields of study such as logic, epistemology, psychology, linguistics and others. Logical and epistemological issues as well as being important in cognitive process are of great significance in science teaching. The main purpose of this paper is to analyze Mario Bunge's meaning theory from an epistemological standpoint and Gérard Vergnaud's theory in the light of cognitive psychology. Relationships to an epistemological view of intricacies are established. Conclusions from this analysis are propounded as theoretically important relating to both teaching and learning processes in science in general and physics in particular.
\end{abstract}

Keywords: Scientific conceptions evolution. Cognitive structure. Science education. Physics teaching.

\footnotetext{
${ }^{1}$ Departamento de Física, Facultad de Ciencias Exactas y Tecnología, Universidad Nacional de Tucumán. $<$ lcudmani@herrera.unt.edu.ar>

${ }^{2}$ Departamento de Física, Facultad de Ciencias Exactas y Tecnología, Universidad Nacional de Tucumán. <mpesa@rectorado.unt.edu.ar>
}

\footnotetext{
1 Avenida Independencia, 1800
}

(4000) Tucumán, Argentina 


\section{Cuestiones epistemológicas introductorias}

\section{La “definición” operacional}

\section{tos científicos.}

Consideraremos en primer término, el problema de la definición de los concep-

Durante veintitrés siglos se ha aceptado la opinión de Aristóteles en el sentido de que la definición es el procedimiento óptimo para asignar significaciones a los signos, pero en la actualidad se toma cada vez mayor conciencia de que es la pertenencia del concepto a un sistema teórico lo que asegura la precisión de su significado.

El apéndice I recoge otros aportes sobre este tema.

En el caso de las ciencias fácticas, un significativo aporte en epistemología, proviene del positivismo.En efecto, una de sus prescripciones metodológicas más significativas se refiere a considerar como válidas, para establecer el significado de los conceptos, sólo a las definiciones operativas. Una de sus figuras más representativa de esta concepción epistemológica, sostiene que "todo concepto, ente o relación, introducida en el lenguaje de la física, debe ser susceptible de una definición operativa, es decir, debe poder ser conocido, determinado y medido, directa o indirectamente, mediante cualquier experiencia o serie de operaciones físicas conceptualmente posibles"(BRIGDMAN, 1927, apud SELVIAGGI, 1983, p. 30).

Newton y los constructores de la Mecánica Clásica, definieron sus términos por sus propiedades. Es clásica su definición de tiempo absoluto: "el tiempo Absoluto, Verdadero y Matemático, por sí mismo y por su naturaleza, discurre uniformemente sin referencia a nada exterior y es lo que se llama duración” (BRIDGMAN, 1927, apud BLANCHÉ, 1972, p. 27). Dentro de esa corriente se atribuye a Einstein, la prescripción de fijar los significados de los conceptos físicos, estableciendo las operaciones físicas (mediciones) mediante las cuales el concepto está determinado, cuando introduce su definición de simultaneidad. Sin embargo el mismo Einstein, en un famoso diálogo que Heisenberg reproduce, relativiza el alcance de esta afirmación (COLOMBO DE CUDMANI, 1994), a limitar las conceptuaciones de la Física a los observables.

Esta propuesta tuvo un gran eco entre los científicos. Roederer (2002), en su excelente texto de Mecánica, afirma que una magnitud física está unívocamente determinada por el proceso de su medición. Coincide así con Bridgman (1927) cuando afirma que el significado del concepto y el conjunto correspondiente de operaciones son sinónimos y que debemos exigir que el conjunto de operaciones equivalentes a un concepto sea único, sino habría en las aplicaciones ambigüedades que no podemos admitir. El significado no sólo está dado por su "definición operacional” sino que además ella es única (ROEDERER, 2002, no adhiere a esta segunda parte). Posiciones similares se encuentran frecuentemente, explícitas o implícitas, en la mayoría de los textos más usuales en la enseñanza universitaria.

Estas posiciones han sido duramente cuestionadas por los epistemólogos contemporáneos. Reflejando las principales críticas a este operativismo, Bunge (1976) (cuya teoría del significado analizaremos más adelante) sostiene que estas "definiciones" no son tales, pues ellas se caracterizan por establecer una relación entre signos cuyo significado está dado por un determinado contexto teórico, el cual les otorga significado por sus interrelaciones. 
Por otra parte, la significación de un signo puede especificarse sobre la base de otros signos (relación signo - signo) o por referencia a objetos no lingüísticos (relación signo hecho). Pero, merece destacarse además que, la definición es una relación signo - signo, en un plano meramente conceptual, en cambio, las relaciones entre objetos lingüísticos y objetos físicos no son definiciones sino que fijan la relación de un signo con su referente

La definición es una de las maneras de precisar la significación de un término, es "una operación puramente conceptual por la cual: se introduce formalmente un nuevo término en algún sistema de signos; se especifica en alguna medida la significación de los términos definientes" (BUNGE, 1976, p. 139).

La definición se inserta en un marco conceptual; en el caso que nos interesa, ese marco conceptual es el del conocimiento científico. El mismo símbolo cambia de significado cuando cambia de contexto. Piénsese en términos como "campo", "trabajo" o "tensión". Obsérvese cómo en todo momento se señala la pertenencia del concepto a un sistema. La sistematización del conocimiento científico, la conexión lógica y epistemológica de sus enunciados, es fundamental para dotar de significado a sus conceptos.

¿Cómo se explica que los científicos hayan adherido con tanta insistencia a estas "definiciones operacionales? Ocurre que ellas establecen una correspondencia entre el signo o el símbolo y su interpretación empírica, establecen correspondencias entre símbolos por un lado y operaciones controladas o sus resultados, por el otro" (BUNGE, 1976, p. 168).

El uso del término "definición operacional" o "definición operativa" favorece la confusión entre significación, que pertenece al campo de la semántica y de la contrastabilidad, que corresponde al campo metodológico. Si se salva esta confusión, entonces el operativismo se reduce a un sano empirismo que exige asegurar una correcta interpretabilidad y contrastabilidad empírica de los términos científicos.

Esta correspondencia se torna sumamente relevante a la hora de convalidar y contrastar las afirmaciones teóricas, conceptuales de la ciencia, con el mundo de los fenómenos reales. Tiene por lo tanto, como ya se señaló, un valor que corresponde al campo de lo metodológico más bien que al semántico.

Debe advertirse también que en la expresión matemática de una ley científica, lo que se somete a operaciones aritméticas son las variables numéricas, los valores de las magnitudes y no su significación conceptual. Del concepto completo, sólo intervienen sus componentes numéricas.

Por otra parte y como ya se dijo, la ciencia emplea también conceptos que no poseen referición operacional precisa. Tal es el caso de "partícula libre", "punto material" o "péndulo matemático", por citar algunos ejemplos.

Insistir en la exclusión de conceptos no operativos del campo de la ciencia, conduce a privar a los científicos de excelentes instrumentos mentales. Como dice Popper, es "matar la gallina de los huevos de oro". En efecto,

la aplicación operacional rigurosa puede servir para atacar especulaciones que pueden finalmente ser fructíferas. Por ejemplo, al final del siglo XIX, el físico Ernst Mach decía que "átomo" es un concepto sin significado físico porque no había forma de observar o medir sus propiedades individuales. Sin embargo, las teorías atómicas desarro- 
lladas en aquel tiempo fueron de gran valor a la ciencia, a pesar de que no podían basarse en definiciones estrictamente operacionales. (POPPER, 1983, p. 263)

$\mathrm{Al}$ respecto, la pretensión del operativismo de que sólo las operaciones de medición pueden suministrar significación a los términos científicos, no reconoce que

las mediciones se llevan a cabo para contrastar determinados enunciados, no para describir significaciones. Es verdad que, al delimitar extensiones, esas operaciones pueden ayudar secundariamente a interpretar ciertos signos [...] y obtenemos, consiguientemente, una captación más rica de su significación [...] pero no nos dará esa significación [...] a menos de poseer ya previamente el concepto y de saber, aunque sea superficialmente, cómo se relaciona con otros conceptos físicos, no seríamos capaces de planear y de realizar la operación de medición. (BUNGE, 1976, p. 172)

Para salvar esta confusión entre lo fáctico y lo semántico, Bunge (1976) propone llamar a estas "definiciones", "refericiones operacionales". Lo que conviene recalcar es que calcular y medir no es definir, pero si es cierto que la comparación de los resultados obtenidos con las mediciones de los valores numéricos de una misma magnitud empleando diferentes refericiones operacionales, la comprobación de una concordancia de esas mediciones, dentro del intervalo de los errores experimentales, si bien no dan el significado del concepto en su total complejidad, ellas enriquecen su comprensión y establecen vínculos que fortalecen a cada uno y a todos los sistemas teóricos en que interviene.

\section{El significado de una proposición}

Como ya vimos anteriormente los enunciados que definen y caracterizan los conceptos de la ciencia, se expresan en forma de proposiciones, por ello es sumamente importante el análisis que se centra sobre su significación. Puesto que los conceptos se caracterizan por su intensión, su referencia y su extensión, la definición prestará atención a estos atributos. La intensión de un concepto es el conjunto de las propiedades que lo caracterizan; la referencia es el conjunto al que denota y la extensión es su dominio de aplicabilidad, de validez.

Estas proposiciones son "constructos", es decir que son objetos conceptuales que satisfacen algún cálculo proposicional (COPI, 1986) y que pueden ser verdaderos o falsos.

Una teoría del significado, rescatando fundamentalmente los aspectos lógicos y epistemológicos, ha sido propuesta por Bunge (1985). Trataremos de presentar una versión simplificada, contextualizada y ejemplificada en el ámbito de la ciencia y su enseñanza.

La definición requiere de términos definientes con significados precisos.

Según esta propuesta, toda proposición se caracteriza en consecuencia, por tener:

* un referente $(\mathbf{R})$ y

$*$ un sentido $(S)$ 
El referente o clase referencia es el conjunto de predicados extra-lógicos que la componen, en un contexto determinado. Esta referencia al contexto no es trivial y es de particular importancia a los fines de este trabajo. Por contexto se entiende un conjunto de proposiciones formadas por conceptos con referentes comunes, referidas a un objeto de estudio que puede o no ser fáctico.

Para clarificar ideas, consideremos un ejemplo sencillo de la cinemática: supuesto un movimiento con velocidad $\mathbf{v}$ y aceleración $\mathbf{a}$, ambas colineales; la velocidad $\mathbf{v}$ al cabo de un cierto tiempo $\mathbf{t}$ de un móvil que se desplaza con aceleración constante es igual a la suma de su velocidad inicial $\mathbf{v}_{\mathbf{o}}$ (para $\mathbf{t}=0$ ), más el producto de $\mathbf{a}$, por el tiempo transcurrido, y puede expresarse como la proposición P:

$$
P: \mathbf{v}=\mathbf{v}_{\mathrm{o}}+\text { a.t }
$$

La clase de referencia de esta proposición $\mathbf{P}$, está dada por el conjunto:

$R_{\mathrm{M}}(\mathbf{P})=(\mathrm{v}, \mathrm{a}, \mathrm{t}$,

En el cual, el subíndice $\mathrm{M}$ hace referencia al contexto.

El sentido se expresaría, en el lenguaje de la lógica simbólica como:

$$
\varphi_{\mathbf{M}}(\mathbf{P})=\left[\mathrm{x} \in \mathbf{M} / \mathrm{x} \leftarrow\left(\mathbf{v}=\mathbf{v}_{\mathrm{o}}+\mathrm{a} . \mathrm{t}\right) \dot{o}\left(\mathbf{v}=\mathbf{v}_{\mathrm{o}}+\mathrm{at}\right) \rightarrow \mathrm{x}\right]
$$

(para toda proposición $\mathrm{x}$ tal que x pertenece a $\mathbf{M}, \mathrm{x}$ implica $\mathrm{a}\left(\mathbf{v}=\mathbf{v}_{\mathrm{o}}+\mathrm{at}\right)$

ó $\left(\mathbf{v}=\mathbf{v}_{\mathbf{o}}+\right.$ at $)$ implica a $\left.\mathbf{x}\right)$

El sentido $\varphi_{\mathbf{M}}(\mathbf{P})$, estará dado por el conjunto de todas las proposiciones que se relacionan lógicamente con la proposición $\mathrm{P}$, en el contexto $\mathbf{M}$. Esto también se expresa como el conjunto formado por todas las proposiciones que implican P o que están implicadas en $\mathrm{P}$, siempre dentro del mismo contexto.

Consideremos las siguientes proposiciones (conocidas relaciones de la mecánica):

- $\mathbf{P}_{1}$ : "Cuando sobre un cuerpo de masa $\mathbf{m}$ actúa una fuerza $\mathbf{f}$, el cuerpo experimenta una aceleración a tal que a es el cociente entre $\mathbf{F}$ y $\mathbf{m}$ ";

O bien,

- $\mathbf{P}_{2}$ : "La aceleración es la derivada de la velocidad respecto al tiempo t";

implican a la preposición $\mathrm{P}$ antes mencionada, $\mathbf{P}$ se deriva de $\mathbf{P}_{1}$ ó $\mathbf{P}_{2}$.

En cambio:

- $\mathbf{P}_{3}$ : "Si la velocidad inicial es cero entonces el tiempo es igual al cociente entre la velocidad al cabo de ese tiempo $\mathbf{v}$ y la aceleración $\mathbf{a " ;}$

o bien,

- $\mathbf{P}_{4}$ : "Si la aceleración es positiva entonces la velocidad $\mathbf{v}$ aumenta con el tiempo";

están implicadas en dicha proposición P.

La referencia o clase de referencia es independiente de las relaciones lógicas que se establecen con $\mathbf{P}$. En cambio el sentido está esencialmente determinado por esas relaciones lógicas. De allí que en el caso del cálculo del sentido, no basta dar el contexto sino que es necesario conocer la estructura lógica del mismo (BUNGE, 1985).

A partir de estas proposiciones, el significado de P estará dado, en este marco teórico por:

$$
\mathrm{S}_{\mathrm{M}}(\mathbf{P})=\varphi_{\mathrm{M}}(\mathbf{P}) \cdot \mathbf{R}_{\mathrm{M}}(\mathbf{P})
$$


Es decir, por "el par ordenado compuesto por su sentido y su referencia, donde sentido es el conjunto de todos los parientes lógicos de P en el contexto M; y referencia es la colección de elementos involucrados en los predicados extralógicos que componen la proposición” (BUNGE, 1985, p. 68).

Este análisis permite, pues, determinar con precisión, cuándo dos proposiciones referidas a un campo factual son iguales o equivalentes, salvando así un problema semántico el cual suele traer muchos problemas y confusiones en la ciencia.

Desde esta perspectiva, dos proposiciones son iguales o equivalentes, es decir tienen el mismo significado sí y solo sí:

- pertenecen al mismo contexto

- tienen igual referente

- tienen igual sentido

Así, la proposición $\mathbf{P}_{1}{ }_{1}$ se enunciaría como:

- $\mathbf{P}_{1}$ : "Para que un cuerpo sufra una aceleración a, debe actuar sobre él una fuerza f; se cumple que a es proporcional a $\mathbf{f}^{\prime \prime}$. En este caso $\mathbf{P}_{1}$ y $\mathbf{P}_{1}{ }_{1}$ son equivalentes.

Otra equivalencia menos trivial sería:

- P”: "El impulso de un cuerpo sobre el cual actúa una fuerza constante $\mathbf{F}$ durante un instante Ät es igual a la variación de la cantidad de movimiento que experimenta el cuerpo durante ese tiempo"

Cuando se trata de poner en claro los elementos que determinan el significado, se pone de manifiesto la importancia del contexto. En ciencia, ese contexto es el marco teórico, es decir la teoría o el modelo dentro del cual se analiza el significado. Si no se explicita, clarifica y delimita ese marco conceptual no puede hablarse de exactitud o de precisión o de objetividad.

Un interesante ejemplo que permite ver cómo depende el significado del contexto de referencia, en particular del sentido de la proposición correspondiente, es el del significado de masa según se tome el contexto de la Mecánica Newtoniana (MN) o el de la Mecánica Relativista (MR). Si bien es cierto que, para bajas velocidades, los valores numéricos coinciden dentro del error experimental, no es menos cierto que el significado del término se ha modificado. La masa, constante característica de un cuerpo, independiente de su estado de movimiento, pasa a ser una función de la velocidad. Algo similar y que ha dado lugar a muchísimas reflexiones e investigaciones vale para el concepto de tiempo en ambas mecánicas.

Popper (1983), hace un interesante análisis relacionado con esta cuestión del significado refiriéndose a la tercera ley de Kepler que relaciona los radios de las órbitas planetarias con sus períodos de rotación: $\mathrm{a}^{3} / \mathrm{T}^{2}=$ constante. En el contexto de la MN, la relación depende de la diferencia entre la masa del Sol y la de cada planeta. Su constancia implicaría, o que todos los planetas tienen masas del mismo orden, lo cual no es cierto, o que la masa de los planetas son todas iguales a cero, lo cual llevaría a una contradicción pues significaría que la fuerza de interacción gravitatoria sería cero. Como se ven, los significados de la proposición son diferentes según el contexto, aún cuando numéricamente se obtengan los mismos resultados, como consecuencia de que las masas planetarias son pequeñas dentro de los errores de medición.

Cuestiones como éstas han originado que algunos epistemólogos contemporáneos (FEYERABEND, 1984; KUHN, 1962) sostengan su tesis de la indecibilidad y la inconmensurabilidad de las teorías científicas, la imposibilidad de evaluar crítica y comparativamente dos teorías rivales. 


\section{Contexto y teoría}

Es pues interesante tratar de clarificar, dentro de esta misma teoría del significado lo que Bunge sostiene respecto de las teorías científicas (BUNGE, 1985).

Según su interpretación, el contexto de una teoría Física está dado por el conjunto de todas las proposiciones que pertenecen a esta teoría.

Una proposición pertenece a una teoría cuando se deduce de sus premisas, es decir de sus axiomas y definiciones. El contexto de una teoría se definiría así por: sus axiomas y definiciones, y todo el conjunto de proposiciones que de ellas se derivan.

Esta línea de razonamiento,

* ¿estaría en contradicción con la posibilidad de generar por medio de la investigación, nuevo conocimiento fáctico dentro de un marco teórico prefijado?

* ¿estaría en contradicción con una concepción de aprendizaje en hélice (COLOMBO DE CUDMANI, SALINAS, PESA, 1991), donde los significados contextuales se acrecientan cada vez que se plantean y se resuelven interrogantes?

* ¿deberíamos entender que el aprendizaje o la investigación implicarán siempre y en todos los casos un cambio de marco conceptual, un cambio de paradigma teórico?

De hecho, esto no es así. En efecto, las afirmaciones de Bunge se refieren a la estructura lógica de una teoría y no a su estructura factual o semántica. A nuestro entender, toda vez que se explicitan nuevas interrelaciones, que se aplica la teoría a una nueva situación problemática, se logra, por un lado, visualizar nuevas concepciones, que a menudo no son obvias ni triviales y por otro lado, se hace necesario introducir informaciones específicas del campo factual en el que se trabaja: condiciones de borde, constantes físicas características del sistema de estudio etc.

Mediante estos mecanismos: aumenta nuestro conocimiento de la realidad física pues se enriquecen los significados; se profundizan los modelos y las explicaciones al crearse nuevos lazos de interrelaciones; se abren nuevos campos de investigación factual y semántica.

Como ejemplo muy significativo de este tipo de conocimiento puede tomarse el proceso deductivo que, en el contexto de la teoría de los campos electromagnéticos, lleva a explicitar la función que rige la propagación de ondas electromagnéticas en el vacío. Se encuentra así un comportamiento no sospechado: la propagación de ondas electromagnéticas cuya velocidad de propagación coincide con la velocidad de propagación de la luz. No puede dudarse del enorme desarrollo de nuevos conocimientos fácticos que de estas conclusiones se desprenden. Permite explicar, además, los fenómenos ópticos desde el punto de vista electromagnético, ampliando enormemente los significados de conceptualizaciones tales como rayo (luz), intensidad, índice de refracción etc.

\section{El significado de los conceptos en la teoría de los campos conceptuales de Vergnaud}

Esta teoría tiene por objeto de estudio del desarrollo y aprendizaje de competencias complejas referidas en particular al aprendizaje de la Matemática pero sin embargo muchos autores (BRAVO 2007; BRAVO, PESA 2005; MOREIRA, 2002, entre otros) han mostrado su 
eficiencia como marco teórico para comprender los aprendizajes científicos y técnicos, con énfasis en los contenidos del conocimiento y la estructura conceptual de los dominios disciplinares. Se procura una mayor comprensión sobre los procesos de construcción del conocimiento, "entendiendo por conocimiento tanto el saber decir como el saber hacer" (VERGNAUD, 1990, p. 133).

En este modelo, se entiende por campos conceptuales a grandes conjuntos de situaciones y problemas, cuyo análisis y tratamiento requiere diversas clases de conceptos, procedimientos y representaciones simbólicas que están interconectados entre sí y probablemente entrelazados durante el proceso de adquisición de conocimiento (VERGNAUD, 1983).

Antes de entrar de lleno a los aportes de la Teoría de Campos Conceptuales (TCC) en lo que se refiere a los significados conceptuales, repasaremos algunos de sus elementos fundamentales.

La TCC analiza la relación "entre conceptos en tanto que conocimiento explícito y las invariantes operatorias implícitas en las conductas de un sujeto en situación; ella explicita también las relaciones entre significante y significado" (VERGNAUD, 1990, p. 133). Aparecen aquí dos conceptos claves para entender la teoría: invariantes operatorios y situación. En el mismo trabajo afirma más adelante que "Un concepto no puede reducirse a su definición" (p. 135). El concepto adquiere su sentido según la situación y los problemas a resolver entendiendo por situación una tarea compleja, o combinación de subtareas, a la cual se enfrenta el sujeto. Refiere a eventos y ocasiones de la realidad, a situaciones cognitivas producidas en la escuela o en la vida diaria que implican acción, ya sea procedimental o declarativa (RODRÍGUEZ PALMERO, MOREIRA, 2004). Al enfrentarla, él se encuentra con objetos, propiedades y relaciones que interactúan de manera compleja en un espacio y en un tiempo dado. Los procesos cognitivos y las respuestas dependen de estas situaciones.

Tal como lo señala Moreira (2002) la TCC es una teoría compleja,

pues involucra la complejidad derivada de la necesidad de abordar en una misma perspectiva teórica, todo el desarrollo de situaciones progresivamente controladas, de conceptos y teoremas necesarios para operar eficientemente en estas situaciones, y de palabras y símbolos que pueden representar eficazmente esos conceptos y operaciones para los estudiante, dependiendo de su desarrollo cognitivo.

Para Vergnaud, "el conocimiento racional es operativo o no es nada". Frente a una situación el sujeto recurre a sus esquemas, concepto eje de toda la teoría, el cual se rescata de la teoría de Piaget, para interpretar los procesos de adaptación: "Se apela a los"esquemas", la organización invariante de la conducta frente a una situación dada. Es en los esquemas donde es necesario investigar los conocimientos-en-acto del sujeto, es decir los elementos cognitivos que permiten a la acción del sujeto ser operativa" (VERGNAUD, 1990, p. 176). El sujeto genera así una secuencia de acciones que no son estereotipos, ya que "no es la conducta lo que es invariante, sino la organización de la conducta". Es una "totalidad organizada que permite generar una clase de conductas diferentes en función de las características particulares de la situación" (VERGNAUD, 1990). "No es un estereotipo, como la secuencia de acciones que depende de los parámetros de la situación, su aplicación implica computaciones, implica cate- 
La evolución de los significados ...

gorías para recoger información relevante y proposiciones de las que se hacen inferencias" (VERGNAUD, 1997).

Los esquemas pueden contener varios ingredientes (VERGNAUD, 1990):

a metas y anticipaciones, que permiten identificar situaciones,

a invariantes operatorios (conceptos-en-acción y teoremas-en acción) mediante los cuales el sujeto puede reconocer los elementos pertinentes de la situación y la información relevante de la misma,

a reglas-de-acción del tipo "si [...] entonces" que permiten generar un secuencia de acciones, a inferencias o razonamientos que se efectúan durante la actividad del sujeto frente a la situación. (BRAVO, 2007, p. 19)

El conjunto de elementos cognitivos de la estructura mental que generan la activación de los esquemas es, en general, implícito, y está constituido por los conceptos-en-acción y los teoremas-en-acción, ellos constituyen los invariantes operatorios, son los conocimientos contenidos en los esquemas, conceptos y teoremas que tienen un status diferente a los conceptos y teoremas científicos. Un concepto-en-acción no es necesariamente un concepto científico ni un teorema-en-acción es necesariamente un principio científico. "En la ciencia, los conceptos y teoremas son explícitos y se puede discutir su pertinencia y validez, y éste no es necesariamente el caso para los invariantes operatorios" (VERGNAUD, 1990).

Los conceptos-en-acción o conocimientos-en-acto, son categorías para obtener información relevante, son los que llevan a buscar la información necesaria para resolver la situación. Pero los conceptos-en-acción no nos permiten razonar: si el razonamiento no tiene la forma de un teorema-en-acción, no se puede resolver el problema o la situación; son proposiciones a partir de las cuales se pueden hacer inferencias, son construcciones mentales más complejas que se pueden considerar verdaderas ó falsas. Los conceptos-en-acción solo pueden ser relevantes o irrelevantes (VERGNAUD, 1994).

Los esquemas representan la organización de la conducta y, en consecuencia, se lo debería considerar como un todo, como una totalidad conformada por invariantes operatorios, reglas-de-acción, anticipaciones e inferencias. La eficacia de estos esquemas depende de la relevancia de los conceptos-en-acción y de la verdad de los teoremas-en-acción.

El conjunto de conceptos-en-acción y teoremas-en-acción constituyen los invariantes operatorios que subyacen a la conducta del sujeto y para hacerlos explícitos, es necesario recurrir a las diferentes formas de representaciones externas tales como gráficas, o signos lingüísticos o gestuales que pongan en evidencia a los mismos. En este proceso de explicitación, los invariantes operatorios se van transformando en conceptos y teoremas explícitos (aunque no necesariamente científicos) a partir de los cuales se puede analizar el conocimiento del sujeto. Por ello resulta importante la representación externa tanto en la investigación como en la enseñanza.

En este contexto, los campos conceptuales son definidos como el conjunto de problemas y situaciones.

Destaca Vergnaud (1983, apud MOREIRA, 2002), tres argumentos principales para la construcción del concepto de campos conceptuales: a) un concepto no se forma dentro de un solo tipo de situaciones: b) una situación no se analiza con un solo concepto c) la 
construcción y apropiación de todas las propiedades de un concepto es un proceso de largo alcance, que se extiende a lo largo de los años.

De estas consideraciones se desprende que la idea fundamental del desarrollo cognitivo es la conceptuación. Por ello se hace necesario generar el marco teórico que permita explicitar qué se entiende por concepto en este modelo.

Vergnaud (1993, 1990, 1983) e Moreira (2002) establecem que un concepto se forma a partir de tres conjuntos: $\boldsymbol{C}=(\mathbf{S}, \mathbf{I}, \mathbf{R})$, tal que:

$\mathbf{S}$ es un conjunto de situaciones que dan sentido al concepto.

- I es un conjunto de invariantes (objetos, propiedades o relaciones sobre los cuales reposa la operacionalidad del concepto, o un conjunto de invariantes operatorios asociados al concepto, o un conjunto de invariantes que pueden ser reconocidos y usados por los sujetos para analizar y dominar las situaciones del primer conjunto.

- $\mathbf{R}$ es un conjunto de representaciones simbólicas (lenguaje natural, gráficos y diagramas, sentencias formales, etc.) que pueden ser usadas para indicar y representar esos invariantes y, consecuentemente, representar las situaciones y los procedimientos para lidiar con ellas. (VERGNAUD, 1990, p. 145)

El primer conjunto es el referente (S), el segundo dará el significado (I) el tercero el significante $(\mathrm{R})$.

Para comprender el significado y el proceso de construcción, estos tres conjuntos no pueden tomarse aisladamente. Ellos deben considerarse en conjunto, como un sistema dinámico en interacción. Es un buen ejemplo de lo que sostiene la Epistemología de la Complejidad: la causalidad no es simple, ni lineal, ni exterior a los objetos "[...] hay una causalidad nueva, que introdujo primero la retroacción cibernética o feedback negativo, en la cual el efecto hace bucle con la causa [...]" (MORIN, 2004, p. 8).

El todo no puede obtenerse simplemente como una suma de partes que se han analizado separadamente. "No hay, en general, biyección entre significantes y significados, ni entre invariantes y situaciones; no puede por tanto reducirse el significado ni a los significantes ni a las situaciones" (VERGNAUD, 1990, p. 146).

De acuerdo con estas concepciones un concepto irá adquiriendo sentido para un sujeto a medida que lo vea interactuar en distintas situaciones y problemas, de las cuales irá abstrayendo las propiedades que considere relevante y reconstruyendo y profundizando su significación. Simultáneamente, cada situación no puede analizarse a través de un único concepto, sino de múltiples conceptos. De allí la razón de estudiar los campos conceptuales y no los conceptos aislados.

En el proceso de construcción del concepto científico adquiere particular importancia el proceso de explicitación:

El proceso de explicitación es difícil, pero los conceptos explícitos y los teoremas explícitos capacitan a los estudiantes para objetivar el conocimiento y discutir su idoneidad y validez [...] No se debate la 
verdad o la falsedad de un enunciado totalmente implícito, y no se identifican los aspectos de la realidad a los que es necesario prestar atención sin la ayuda de palabras, de enunciados, de símbolos y de signos. El uso de significantes explícitos es indispensable para la conceptuación. (VERGNAUD, 1994)

Un concepto no llega a ser totalmente un concepto si no se hace explícito (conjunto R).

El sentido surge de la relación entre el sujeto, a través de sus conocimientos, con las situaciones y con los significantes (VERGNAUD, 1990, p. 158). "Más precisamente, al sentido lo constituyen no sólo los esquemas evocados por el sujeto ante una situación o por un significante sino que surge de la relación entre lo que cada individuo es capaz de hacer y/o de expresar frente a ciertas situaciones en función de los esquemas de que dispone" (ESCUDERO, MOREIRA, CABALLERO, 2004). Por ejemplo, el sentido de onda electromagnética para un determinado sujeto es lo que es capaz de hacer y/o de expresar con los esquemas que activa al enfrentar las situaciones que le proporcionan y que implican este concepto. Es también lo que es capaz de hacer y expresar con los esquemas disponibles al operar con símbolos numéricos, algebraicos, representaciones gráficas y língüísticas que representan a la onda electromagnética.

\section{Algunos comentarios e implicaciones para la enseñanza de la ciencia}

Pese a haber partido de marcos referenciales y de objetivos diferentes, consideramos que los dos modelos presentados, el de Bunge y el de Vergnaud, hacen aportes interesantes para la enseñanza de la ciencia. Sus visiones se complementan con sus importantes aportes, uno desde lo lógico y epistémico y el otro desde lo psicológico cognitivo.

Consideramos destacable las coincidencias respecto a la complejidad de la cuestión, en ambos casos muy apartada de la concepción reduccionista del positivismo y sus "definiciones operacionales".

Para Bunge, el sentido del concepto está dado por la intersección de todos los haces de relaciones de implicación y de implicancia en las que el concepto interviene, en el contexto de una teoría o de un marco conceptual.

Este concepto de contexto o marco conceptual, se enriquece en Vergnaud con la idea de invariantes operatorios, conceptos-en-acción y teoremas-en-acción, que el sujeto activa frente a un problema concreto. En ambos casos, si bien desde puntos de vista diferentes, el referente es una "realidad" objeto del conocimiento.

Estas interpretaciones del contexto se condicen perfectamente con una concepción estructuralista del aprendizaje. No sólo los hechos carecen de significación por sí mismo y deben ser interpretados a partir de ciertas conceptualizaciones, también los conceptos y proposiciones adquieren significados más objetivos y precisos dentro de un contexto lógico, epistemológico o psicológico.

Contra la concepción basada en un inductivismo ingenuo según la cual los significados quedan claramente determinados por una definición operativa a partir de la observación, como meras generalizaciones de los hechos, lo que aquí se sostiene es que los enunciados 
observables usan siempre el contexto de alguna teoría, explícita o no. Estos enunciados y conceptuaciones serán tan precisos como lo sea el marco conceptual o teórico que se tome como referencia.

Para definir una magnitud física puede usarse, dentro del mismo contexto, diferentes relaciones; toda relación funcional en que aparezca la magnitud proporcionará una forma operacional para definirla. No existe pues, una definición completa de un concepto físico o, en general, científico. Pero desde un punto de vista psicológico, será la situación problemática la que desencadene a qué conceptuaciones, a qué invariantes operatorios recurrirá el sujeto.

En el estado actual de la investigación científica ninguna proposición aislada tiene un significado claro,

ella se inserta en estructuras y las partes de esas estructuras toman su significado los unos de los otros y del todo del cual forman parte... es imposible discutir el trabajo sin considerar la fuerza y ésta sin considerar la cantidad de movimiento [...] si queremos evitar que la ciencia sea incomprendida o malentendida debemos transmitirla como un relato de la investigación para hacer perceptible a los estudiantes la naturaleza de la estructura que hizo posible esa ciencia. (SCHWAB, 1973)

En esta misma línea, Morin (1999; GALLEGOS, 2000) plantea en su epistemología de la "Complejidad" (y por cierto este problema de los significados posee todas las características de un sistema complejo), que

[...] situar a un acontecimiento en su contexto, incita a ver como éste modifica al contexto o como le da una luz diferente. Un pensamiento como éste se vuelve inseparable del pensamiento de los complejo, pues no basta con inscribir todas las cosas y los hechos en un "marco" u horizonte. Se trata de buscar siempre las relaciones e interretro-acciones entre todo fenómeno y su contexto, las relaciones recíprocas entre el todo y las partes, cómo una modificación local repercute sobre el todo y cómo una modificación del todo repercute sobre las partes. (MORIN, 1999, p. 27)

Por otro lado una misma magnitud, por ejemplo la fuerza, tendrá definiciones diferentes en distintos contextos teóricos, tanto más precisas cuanto más precisos sean esos contextos. Así, para Aristóteles la fuerza "es igual a la resistencia multiplicada por la velocidad", para Newton "es igual a la masa multiplicada por la aceleración", para Einstein "es igual a la variación temporal del impulso", para Eherenfest "la fuerza media es igual al valor medio de la variación temporal del impulso", para Bohm "la fuerza exterior más la fuerza cuántica es igual a la variación temporal del impulso oculto". El aprendizaje del concepto se va profundizando a medida que se lo reestructura a través de los correspondientes cambios conceptuales implicados en el paso de una teoría a otra más profunda.

Otro aporte significativo se refiere a proporcionar un valioso marco para interpretar 
los llamados preconceptos, o conceptos "erróneos" o "errores conceptuales". Puesto que el significado está dado por el contexto, es claro que las interpretaciones de las situaciones a problemáticas y sus soluciones dependen fundamentalmente del marco teórico de referencia (BUNGE) o del conjunto de invariantes operatorios asociados al concepto, que pueden ser reconocidos y usados por los sujetos para analizar y dominar la situación problemática a resolver. Por ello, parecería que las denominaciones más acertadas para estas dificultades en el aprendizaje de las ciencias serían las de "concepciones contextualmente erróneas" (MOREIRA, NOVAK, 1988) o las de "concepciones alternativas" (POSNER, 1982) donde se hace explicita referencia a que el "error" es consecuencia de haber analizado la situación desde un contexto diferente (HEWSON, HEWSON, 1983).

Aún dentro de un contexto dado, el conocimiento, la comprensión, el significado se enriquecen permanentemente en la medida en que se encaren soluciones a situaciones problemáticas nuevas. Se hace necesario explicitar nuevas interrelaciones. Se deben determinar nuevos items de información fáctica, etc. Todo lo cual además de permitir dar respuesta al problema, se traduce en un aumento y profundización de los significados.

El concepto densidad, por ejemplo, es mucho más rico y complejo que una receta para medirla como "divida la masa por el volumen" En el contexto de la mecánica el significado de densidad estará dado por la intersección de todas las relaciones en que este concepto aparezca (CUDMANI, 1991). Está claro que el concepto al que nos referimos se enriquecerá y se reconstruirá en la medida en que se analicen distintas relaciones en las que interviene, referidas a diferentes situaciones fenomenológicas. Los docentes sabemos cuánto cuesta a los alumnos discriminar entre la densidad y la viscosidad de un cuerpo, o comprender la importancia de la densidad en la propagación de ondas o en fenómenos de flotación, para citar solo algunos ejemplos.

Las definiciones de la ciencia son, como el conocimiento científico, perfectibles. Justamente porque presuponen tanto teoría como información empírica, nueva información o cambios teoréticos pueden modificarla. Eso mismo ocurre con todos los conceptos científicos; aún aquellos que no cambian su significado, lo precisan cada vez más a medida que intervienen en más (o más profundos) sistemas teóricos.

Se rescata así, en ambos enfoques, el gran valor que tiene para el aprendizaje enfrentar al estudiante con situaciones problemáticas de toda índole o incentivarlo para que él mismo se las plantee, tanto en situaciones experimentales como teóricas.

De la construcción de Bunge, puesto que fórmulas, cálculos, algoritmos matemáticos son signos que simbolizan conceptos científicos, resulta que la exactitud y precisión de las conclusiones depende del rigor y exactitud cualitativa, puesto que el significado y la interpretación de estas conclusiones sólo cobran sentido, rigor y precisión, dentro de un contexto que debe ser claro y explicito. Sin esta precisión cualitativa, la precisión cuantitativa carece de referente y sentido claros y precisos, y por lo tanto de significado claro y preciso.

Por otro lado, la construcción de Vergnaud, arroja luz sobre el carácter idiosincrático de estas conceptuaciones. Sólo la argumentación y la búsqueda de consenso con otros sujetos permitirán ir construyendo el significado que a los científicos les llevó años, a veces siglos, construir.

Si bien la ciencia ha aportado muchas certezas nos ha revelado múltiples campos de incertidumbre, 
el pensamiento complejo se crea y se recrea en el mismo caminar. El pensamiento complejo, confrontado a la pura simplificación, es un pensamiento que postula la dialógica y la recursividad como sus principios más pertinentes y donde se reconoce a la incertidumbre como uno de los legados de la ciencia contemporánea. (ACOSTA RODRÍGUEZ, 2006, p. 126)

Surge así la necesidad de enseñar y aprender estrategias que permitan afrontar los riesgos, lo inesperado, lo incierto y modificar el desarrollo de esquemas y marcos conceptuales en función de las nuevas informaciones adquiridas

Profesores y alumnos deberán enfrentarse al reto de tener que reelaborar un significado que creían conocido, cuando este se involucra en una situación nueva (VERGNAUD) o en nuevas relaciones de implicancias e implicaciones. Frente al pensamiento lineal y reduccionista y a la certeza de la definición operacional, se genera un ámbito de ambigüedad que debe ser debidamente asumido.

\section{Conclusiones}

A partir del estudio de un aspecto específico del conocimiento científico este trabajo pone de manifiesto los importantes aportes que la reflexión epistemológica y psicológica puede hacer a la enseñanza y a la investigación en ciencia, no ya como un análisis desde afuera sino como parte constitutiva de la construcción del conocimiento científico.

A nuestro entender este análisis a partir de la teoría del significado, proporciona elementos válidos para comprender la importancia de lo contextual en relación con el significado. Proporciona un marco de referencia que permite comprender, dentro de la estructura lógica de la teoría científica y la psicológica de los invariantes operatorios, el dinamismo del aprendizaje y la investigación.

Los significados se modifican y se perfeccionan cada vez que se explicitan nuevas relaciones de implicancia, se desarrollan nuevos subsistemas, se cierran nuevos lazos e interrelaciones que acrecienten la comprensión de concepto y proposiciones. El sistema funciona, en lo que hace a la significación como un sistema de vasos comunicante donde el aumento de comprensión de alguno de sus componentes enriquece la comprensión de todos los demás elementos. Este proceso responde bien al modelo del "bucle recursivo" que es "[...] un bucle generador en el que los productos y los efectos son en sí mismo productores y causantes de lo que los produce" (MORIN, 1999)

Se pone en evidencia una vez más que es mejor medida de nuestro aprendizaje la cantidad de problemas y cuestiones que somos capaces de plantear que la cantidad de problemas y cuestiones que somos capaces de resolver. 
La evolución de los significados ...

\section{Referências}

ACOSTA RODRÍGUEZ, M. El aprendizaje visto como construcción social, la perspectiva vigotskiana vista desde la complejidad. Revista Ciencias de la Educación, España, v. 1, n. 27, p. 123-34, 2006.

BRAVO, S. Concepciones de los estudiantes referidas a los fenómenos ondulatorios. 2006. Tesis (Maestría) - Facultad de Ciencias Exactas y Tecnología, Universidad Nacional de Tucumán, Argentina, 2006.

BLANCHÉ, R. El método experimental y la filosofía de la física. México: Fondo de Cultura Económica, 1972.

BRAVO, S.; PESA, M. La construcción de representaciones sobre movimiento ondulatorio: una interpretación de la teoría de campos conceptuales de Vergnaud y la teoría de modelos mentales de Jonson-Laird. Revista de Enseñanza de la Física, Córdoba, v. 18, n. 2, p. 25-42, 2005.

BRIDGMAN, P. N. The logic of modern Physics. New Cork: Mac Millan Ed., 1927.

BUNGE, M. Epistemología. Barcelona: Ariel, 1985.

La investigación científica. Barcelona: Ariel, 1976.

COLOMBO DE CUDMANI., L. Einstein y Heisenberg. Rev. Enseñanza de la Física, Córdoba, v. 6, n. 2, 1993.

.; SALINAS, J.; PESA, M. La generación autónoma de conflictos cognoscitivos para favorecer cambios de paradigmas en el aprendizaje de la Física. Enseñanza de las Ciencias, Espanha, v. 9, n. 3, p. 237-42, 1991.

COPI, M. Introducción a la lógica. Buenos Aires: EUDEBA, 1986. [manuales].

ESCUDERO, C.; MOREIRA, M. A.; CABALLERO, C. Teoremas en acción y conceptos en acción en clases de física introductoria secundaria. In: MOREIRA, M. A. (Ed.). La teoría de los campos conceptuales de Vergnaud, la enseñanza de las ciencias y la investigación en el área. Porto Alegre: Instituto de Física da UFRGS, 2004. p. 93-116.

FEYERABEND, F. K. Contra el método. Argentina: Edit Orbis, 1984.

GALLEGOS, M. La Epistemol de la Complejidad como recurso para la educación. Argentina: Facultad de Sociología, Universidad Nacional de Rosario, 2000.

HEWSON, M.; HEWSON, P. Effect of instruction using students' prior knowledge and conceptual change strategies. Journal of Research in Science Teaching, v. 20, n. 8, 1983.

KUHN, T. The structure of scientific revolutions. USA: University of Chicago Press, 1962.

MOREIRA, M. A. A teoria dos campos conceptuais de Vergnaud, ensino de ciencias e a pesquisa nesta área. Investigações em Ensino de Ciências, Porto Alegre, v. 7, n. 1, 2002. 
Cudmani, L. C.; Pesa, M. A.

MOREIRA, M. A.; NOVAK, J. D. Investigación en enseñanza de las Ciencias en la Universidad de Cornell. Enseñanza de las Ciencias, Barcelona, v. 6, n. 1, p. 3-18, 1988.

MORIN, E. La epistemología de la complejidad correspondiente a la intelligence de la complexitè. Paris: Ed L'Harmattan, 2004.

La cabeza bien puesta: repensar la reforma, reformar el pensamiento. Buenos Aires: Nueva Vision, 1999.

POPPER, K. El conocimiento objetivo. Madri: Technos, 1983.

POSNER, G. J. et al. Accomodation of scientific conceptions: towars a theory of conceptual change. Science Education, n. 66, p. 221-7, 1982.

SCHWAB, J. Problemas, tópicos y puntos de discusión. In: ELAM, E. La educación y la estructura del conocimiento. Buenos Aires: El Ateneo, 1973. (compilación)

ROEDERER, J. G. Mecánica elemental. Buenos Aires: EUDEBA, 2002.

RODRÍGUEZ PALMERO, M. L.; MOREIRA, M. A. La teoria de los campos conceptuales de Gérard Vergnaud. In: MOREIRA, M. A. (Ed.). La teoría de los campos conceptuales de Vergnaud, la enseñanza de las ciencias y la investigación en el área. Porto Alegre: Instituto de Física da UFRGS, 2004. p. 7-40.

SELVIAGGI, F. Orientaciones actuales de la Física. Buenos Aires: Troquel, 1983.

VERGNAUD, G. Quelques problèmes theóriques de la didactique a propos d'un example: les estructures additives. In: ATELIER INTERNACIONAL D'ETE: RECHERCHE EN DIDACTIQUE DE LA PHYSIQUE, 1983, France. Anais... France, 1983.

. The nature of mathematical concepts. In: NUNES, T.; BRYANT, P. (Eds.). Learning and Teaching Mathematics: An International Perspective. Hove, UK: Psychology Press. 1997. p. 5-28.

. Multiplicative conceptual field: what and why? In: HAREL, G.; COUFREY, J.

(Eds.). The development of multiplicative reasoning in the learning of mathematics. USA: State University of New York Press, 1994. p. 41-59

. Teoria dos campos conceptuais. In: SEMINARIO INTERNATIONAL DE EDUCAÇÃO MATEMÁTICA DO RIO DE JANEIRO, 1., 1993, Rio de Janeiro. Anais... Rio de Janeiro, 1993. p. 1-26.

La théorie des champs conceptuels. Recherches en Didactique des Mathématique, v. 10, n. 23, p. 133-70, 1990.

Artigo recebido em dezembro de 2007 e aprovado em junho de 2008. 